\title{
Resonant Laser Excitation of Molecular Wires
}

\author{
Sigmund Kohler, Jörg Lehmann, Sébastien Camalet, and Peter Hänggi* \\ Institut für Physik, Universität Augsburg, Universitätsstraße 1, D-86135 Augsburg, Germany
}

(Received 17 September 2002 and in revised form 30 October 2002)

\begin{abstract}
We investigate the influence of external laser excitations on the average current through bridged molecules. For the computation of the current, we use a numerically very efficient formalism that is based on the Floquet solutions of the time-dependent molecule Hamiltonian. It is found that the current as a function of the laser frequency exhibits characteristic peaks originating from resonant excitations of electrons to bridge levels that are unoccupied in the absence of radiation. The electric current through the molecule can exhibit a drastic enhancement by several orders of magnitude.
\end{abstract}

\section{INTRODUCTION}

In a seminal work, ${ }^{1}$ Aviram and Ratner proposed almost thirty years ago to build elements of electronic circuits - in their case, a rectifier-with single molecules. Today their vision is starting to become reality, and the experimental and theoretical study of such systems enjoys vivid activity. ${ }^{2-4}$ Recent experimental progress has enabled reproducible measurements ${ }^{5,6}$ of weak tunneling currents through molecules that are coupled by chemisorbed thiol groups to the gold surface of external leads. A necessary ingredient for future technological applications will be the possibility to control the tunneling current through the molecule.

Typical energy scales in molecules are in the optical and the infrared regime, where basically all of today's lasers operate. Hence, lasers represent a natural possibility to control atoms or molecules and also currents through them. It is, for example, possible to induce by the laser field an oscillating current in the molecule that under certain asymmetry conditions is rectified by the molecule, resulting in directed electron transport even in the absence of any applied voltage. ${ }^{7,8}$ Another theoretically predicted effect is current suppression by the laser field, ${ }^{9}$ which offers the possibility to control and switch the electron transport by light. Since the frequencies considered lie below typical plasma frequencies of metals, the laser light will be reflected at the metal surface, i.e., it does not penetrate the leads. Consequently, we do not expect major changes of the leads' bulk propertiesin particular, each lead remains close to equilibrium. Thus, to a good approximation, it is sufficient to consider the influence of the driving solely in the molecule Hamiltonian. In addition, the energy of infrared light quanta is by far smaller than the work function of a common metal, which is of the order of $5 \mathrm{eV}$. This prevents the generation of a photo current that would dominate the effects discussed below.

Recent theoretical descriptions of the molecular conductivity in non-driven situations are based on a scattering approach, ${ }^{10,11}$ or assume that the underlying transport mechanism is an electron transfer reaction from the donor to the acceptor site and that the conductivity can be derived from the corresponding reaction rates. ${ }^{3}$ It has been demonstrated that both approaches yield essentially identical results in a large parameter regime. ${ }^{12}$ Within a high-temperature limit, electron transport on the wire can be described by inelastic hopping events. $^{13,14}$

*Author to whom correspondence should be addressed. E-mail: hanggi@physik.uni-augsburg.de

$$
\text { Israel Journal of Chemistry } \quad \text { Vol.42 } 2002 \quad \text { pp. 135-141 }
$$


Atoms and molecules in strong oscillating fields have been widely studied within a Floquet formalism. ${ }^{15,16}$ This suggests utilizing the tools that have been acquired in that area, thus developing a transport formalism that combines Floquet theory for a driven molecule with the many-particle description of transport through a system that is coupled to ideal leads. ${ }^{7-9}$ Such an approach is devised much in the spirit of the FloquetMarkov theory ${ }^{17,18}$ for driven dissipative quantum systems.

A rather heuristic approach to the problem of driven molecular wires has been introduced in ref 19. It is based on the assumption that each sideband of a Floquet state contributes as an independent conduction channel to the average current, and that each contribution can be calculated from a current formula that has been derived for the time-independent situation. As a consequence, this approach does not allow a consistent treatment of the coherences between different Floquet states or of the coherence between different sidebands within a Floquet state.

\section{FLOQUET TREATMENT OF ELECTRON TRANSPORT}

The entire system of the wire in the laser field, the leads, and the molecule-lead coupling, as sketched in Fig. 1, is described by the Hamiltonian

$$
H(t)=H_{\text {wire }}(t)+H_{\text {leads }}+H_{\text {wire-leads }}
$$

The wire is modeled by $N$ atomic orbitals $|n\rangle, n=1$, ..., $N$, which are in a tight-binding description, coupled by hopping matrix elements. Then, the corresponding Hamiltonian for the electrons on the wire, in a second quantized form, is

$$
H_{\text {wire }}(t)=\sum_{n, n^{\prime}} H_{n n^{\prime}}(t) c_{n}^{\dagger} c_{n^{\prime}}
$$

where the fermionic operators $c_{n}, c_{n}^{\dagger}$ annihilate and create, respectively, an electron in the atomic orbital $|n\rangle$ and obey the anti-commutation relation $\left[c_{n}, c_{n^{\prime}}^{\dagger}\right]_{+}=\delta_{n, n^{\prime}}$. The influence of the laser field is given by a periodic time-dependence of the on-site energies yielding a single particle Hamiltonian of the structure $H_{n n^{\prime}}(t)=$ $H_{n n^{\prime}}(t+\mathcal{T})$, where $\mathcal{T}=2 \pi / \Omega$ is determined by the angular frequency $\Omega$ of the laser field.

The orbitals $|1\rangle$ and $|N\rangle$ at the left and the right ends of the molecule, which we shall term donor and acceptor, respectively, are coupled to ideal leads (cf. Fig. 1) by the tunneling Hamiltonians

$$
H_{\text {wire-leads }}=\sum_{q}\left(V_{q L} c_{q L}^{\dagger} c_{1}+V_{q R} c_{q R}^{\dagger} c_{N}\right)+\text { H.c. }
$$

The operator $c_{q L}\left(c_{q R}\right)$ annihilates an electron in state $q L(q R)$ on the left (right) lead. The leads are modeled as non-interacting electrons with the Hamiltonian

$$
H_{\text {leads }}=\sum_{q}\left(\epsilon_{q L} c_{q L}^{\dagger} c_{q L}+\epsilon_{q R} c_{q R}^{\dagger} c_{q R}\right)
$$

where $\epsilon_{q L}$ is the single particle energy of state $q L$ and correspondingly for the right lead. As discussed above, the leads can be described by a grand-canonical ensemble of electrons, i.e., by a density matrix

$$
\varrho_{\text {leads,eq }} \propto \exp \left[-\left(H_{\text {leads }}-\mu_{L} N_{L}-\mu_{R} N_{R}\right) / k_{B} T\right]
$$

where $\mu_{L / R}$ are the electrochemical potentials and $N_{L / R}=$ $\Sigma_{q} c_{q L / R}^{\dagger} c_{q L / R}$ the electron numbers in the left/right lead. As a consequence, the only non-trivial expectation values of lead operators are

$$
\left\langle c_{q L}^{\dagger} c_{q L}\right\rangle=f\left(\epsilon_{q L}-\mu_{L}\right)
$$

Here, $f(x)=\left(1+e^{x / k_{B} T}\right)^{-1}$ denotes the Fermi function.

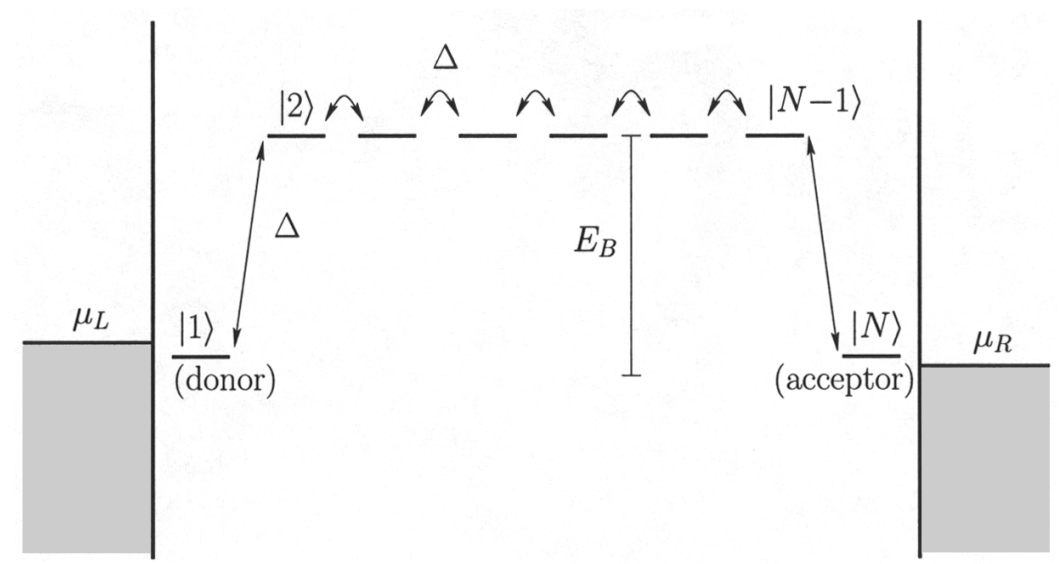

Fig. 1. Level structure of a molecular wire with $N=8$ atomic sites, which is attached to two leads. 


\subsection{Perturbation Theory}

While the leads and the wire, including the driving, will be treated exactly, we take into account the wirelead Hamiltonian as a perturbation. Starting from the Liouville-von Neumann equation $i \hbar \dot{\varrho}(t)=[H(t), \varrho(t)]$, together with the factorizing initial condition $\varrho\left(t_{0}\right)=$ $\varrho_{\text {wire }}\left(t_{0}\right) \otimes \varrho_{\text {leads,eq }}$, we derive by standard techniques an approximate equation of motion for the total density operator $\varrho(t)$. This is most conveniently achieved in the interaction picture with respect to the uncoupled dynamics, where the Liouville-von Neumann equation is

$$
i \hbar \frac{d}{d t} \tilde{\varrho}\left(t, t_{0}\right)=\left[\widetilde{H}_{\text {wire-leads }}\left(t, t_{0}\right), \tilde{\varrho}\left(t, t_{0}\right)\right]
$$

The tilde $(\sim)$ denotes the corresponding interaction picture operators, $\widetilde{X}\left(t, t^{\prime}\right)=U_{0}^{\dagger}\left(t, t^{\prime}\right) X(t) U_{0}\left(t, t^{\prime}\right)$, where the propagator of the wire and the lead in the absence of the lead-wire coupling is given by the timeordered product

$$
\begin{gathered}
U_{0}\left(t, t^{\prime}\right)= \\
\overleftarrow{T} \exp \left(-\frac{i}{\hbar} \int_{t^{\prime}}^{t} d t^{\prime \prime}\left[H_{\text {wire }}\left(t^{\prime \prime}\right)+H_{\text {leads }}\right]\right)
\end{gathered}
$$

Equation 7 is equivalent to the following integral equation:

$$
\begin{gathered}
\tilde{\varrho}\left(t, t_{0}\right)= \\
\tilde{\varrho}\left(t_{0}, t_{0}\right)-\frac{i}{\hbar} \int_{t_{0}}^{t} d t^{\prime}\left[\widetilde{H}_{\text {wire-leads }}\left(t^{\prime}, t_{0}\right), \tilde{\varrho}\left(t^{\prime}, t_{0}\right)\right]
\end{gathered}
$$

We reinsert this expression into the differential equation (eq 7) and use that to zeroth order in the molecule-lead coupling the interaction-picture density operator does not change with time, $\tilde{\varrho}\left(t-\tau, t_{0}\right) \approx \tilde{\varrho}\left(t, t_{0}\right)$. A transformation back to the Schrödinger picture results in the following approximate equation of motion for the total density operator ${ }^{7,8}$

$$
\begin{aligned}
& \dot{\varrho}(t)=-\frac{i}{\hbar}\left[H_{\text {wire }}(t)+H_{\text {leads }}, \varrho(t)\right] \\
& -\frac{1}{\hbar^{2}} \int_{0}^{\infty} d \tau\left[H_{\text {wire-leads }},\left[\widetilde{H}_{\text {wire-leads }}(t-\tau, t), \varrho(t)\right]\right] \\
& -\frac{i}{\hbar}\left[H_{\text {wire-leads }}, U_{0}\left(t, t_{0}\right) \varrho\left(t_{0}\right) U_{0}^{\dagger}\left(t, t_{0}\right)\right]
\end{aligned}
$$

Since we only consider asymptotic times $t-t_{0} \rightarrow \infty$, we have set the upper limit in the integral to infinity. The third term in eq 10 stems from the initial condition at $t_{0}$ in the integrated form (eq 9) of the Liouville-von Neumann equation. For the chosen factorizing initial condition, it will not contribute to the expectation values calculated below.
The net (incoming minus outgoing) current through the left contact is given by the negative time derivative of the electron number in the left lead, multiplied by the electron charge $-e$, i.e.,

$$
I_{L}(t)=e \operatorname{tr}\left[\dot{\varrho}(t) N_{L}\right]
$$

We insert $\dot{\varrho}(t)$ from eq 10 and obtain an expression that depends on the density of states in the leads times their coupling strength to the connected sites. At this stage, it is convenient to introduce the spectral density of the lead-wire coupling

$$
\Gamma_{L / R}(\epsilon)=\frac{2 \pi}{\hbar} \sum_{q}\left|V_{q L / R}\right|^{2} \delta\left(\epsilon-\epsilon_{q L / R}\right)
$$

which fully describes the leads' influence. If the lead states are dense, $\Gamma_{L / R}(\epsilon)$ becomes a continuous function. Because we are mainly interested in the behavior of the molecule and not in the details of the lead-wire coupling, we assume that the conduction bandwidth of the leads is much larger than all remaining relevant energy scales. Consequently, we approximate in the socalled wide-band limit the functions $\Gamma_{L / R}(\epsilon)$ by the constant values $\Gamma_{L / R}$. After some algebra, we find for the time-dependent net electrical current through the left contact the expression

$$
\begin{aligned}
I_{L}(t)= & \frac{e \Gamma_{L}}{\pi \hbar} \operatorname{Re} \int_{0}^{\infty} d \tau \int d \epsilon e^{i \epsilon \tau / \hbar} \\
& \left\{\left\langle\tilde{c}_{1}^{\dagger}(t-\tau, t) c_{1}\right\rangle\right. \\
& \left.-\left[c_{1}, \tilde{c}_{1}^{\dagger}(t-\tau, t)\right]_{+} f\left(\epsilon-\mu_{L}\right)\right\}
\end{aligned}
$$

and, correspondingly, for the current through the contact on the right-hand side. Here, we made the assumption that the leads are at all times well described by the density operator (eq 5). Note that the anti-commutator $\left[c_{1}, \tilde{c}_{1}^{\dagger}(t-\tau, t)\right]_{+}$is in fact a $c$-number. Like the expectation value $\left\langle c_{1}^{\dagger}(t-\tau, t) c_{1}\right\rangle$, it depends on the dynamics of the isolated wire and is influenced by the external driving. The first contribution of the $\epsilon$-integral in eq 13 is readily evaluated to yield an expression proportional to $\delta(\tau)$. Thus, this term becomes local in time and is $e \Gamma_{L}\left\langle c_{1}^{\dagger} c_{1}\right\rangle$.

\subsection{Floquet Decomposition}

Let us next focus on the single-particle dynamics of the driven molecule decoupled from the leads. Since its Hamiltonian is periodic in time, $H_{n n^{\prime}}(t)=H_{n n^{\prime}}(t+\mathcal{T})$, we can solve the corresponding time-dependent Schrödinger equation within a Floquet approach. This means that we make use of the fact that there exists a complete set of solutions of the form ${ }^{16,20-23}$ 


$$
\begin{aligned}
& \left|\Psi_{\alpha}(t)\right\rangle=e^{-i \epsilon_{\alpha} t / \hbar}\left|\Phi_{\alpha}(t)\right\rangle, \\
& \left|\Phi_{\alpha}(t)\right\rangle=\left|\Phi_{\alpha}(t+\mathcal{T})\right\rangle
\end{aligned}
$$

with the quasi-energies $\epsilon_{\alpha}$. Since the so-called Floquet modes $\left|\Phi_{\alpha}(t)\right\rangle$ obey the time-periodicity of the driving field, they can be decomposed into the Fourier series

$$
\left|\Phi_{\alpha}(t)\right\rangle=\sum_{k} e^{-i k \Omega t}\left|\Phi_{\alpha, k}\right\rangle
$$

This implies that the quasi-energies $\epsilon_{\alpha}$ come in classes,

$$
\epsilon_{\alpha, k}=\epsilon_{\alpha}+k \hbar \Omega, \quad k=0, \pm 1, \pm 2, \ldots
$$

all members of which represent the same solution of the Schrödinger equation. Therefore, the quasi-energy spectrum can be reduced to a single "Brillouin zone" $-\hbar \Omega / 2 \leq \epsilon<\hbar \Omega / 2$. In turn, all physical quantities that are computed within a Floquet formalism are independent of the choice of a specific class member. Thus, a consistent description must obey the so-called class invariance, i.e., it must be invariant under the substitution of one or several Floquet states by equivalent ones,

$$
\epsilon_{\alpha},\left|\Phi_{\alpha}(t)\right\rangle \longrightarrow \epsilon_{\alpha}+k_{\alpha} \hbar \Omega, e^{i k_{\alpha} \Omega t}\left|\Phi_{\alpha}(t)\right\rangle
$$

where $k_{1}, \ldots, k_{N}$ are integers. In the Fourier decomposition (eq 15), the prefactor $\exp \left(i k_{\alpha} \Omega t\right)$ corresponds to a shift of the side band index so that the class invariance can be expressed equivalently as

$$
\epsilon_{\alpha},\left|\Phi_{\alpha, k}\right\rangle \longrightarrow \epsilon_{\alpha}+k_{\alpha} \hbar \Omega,\left|\Phi_{\alpha, k+k_{\alpha}}\right\rangle
$$

Floquet states and quasienergies can be obtained from the quasienergy equation ${ }^{15,16,20-23}$

$$
\left(\sum_{n, n^{\prime}}|n\rangle H_{n n^{\prime}}(t)\left\langle n^{\prime}\right|-i \hbar \frac{d}{d t}\right)\left|\Phi_{\alpha}(t)\right\rangle=\epsilon_{\alpha}\left|\Phi_{\alpha}(t)\right\rangle
$$

A wealth of methods for the solution of this eigenvalue problem can be found in the literature. For an overview, we refer the reader to the reviews in refs 16,23 , and references therein.

As the equivalent of the one-particle Floquet states $\left|\Phi_{\alpha}(t)\right\rangle$, we define a Floquet picture for the fermionic creation and annihilation operators $c_{n}^{\dagger}, c_{n}$ by the timedependent transformation

$$
c_{\alpha}(t)=\sum_{n}\left\langle\Phi_{\alpha}(t) \mid n\right\rangle c_{n}
$$

The inverse transformation

$$
c_{n}=\sum_{\alpha}\left\langle n \mid \Phi_{\alpha}(t)\right\rangle c_{\alpha}(t)
$$

follows from the mutual orthogonality and the completeness of the Floquet states at equal times. ${ }^{16,23}$ Note that the right-hand side of eq 21 becomes $t$-independent after the summation. The operators $c_{\alpha}(t)$ are constructed in such a way that the time-dependences of the interaction picture operators $\tilde{\mathrm{c}}_{\alpha}(t-\tau, t)$ separate, which will turn out to be crucial for the further analysis. Indeed, one can easily verify the relation

$$
\begin{aligned}
\tilde{c}_{\alpha}(t-\tau, t) & =U_{0}^{\dagger}(t-\tau, t) c_{\alpha}(t-\tau) U_{0}(t-\tau, t) \\
& =e^{i \epsilon_{\alpha} \tau / \hbar} c_{\alpha}(t)
\end{aligned}
$$

by differentiating the definition in the first line with respect to $\tau$ and using that $\left|\Phi_{\alpha}(t)\right\rangle$ is a solution of the eigenvalue eq 19. The fact that the initial condition $\tilde{c}_{\alpha}(t, t)=c_{\alpha}(t)$ is fulfilled completes the proof. The corresponding expression for the interaction picture operator in the on-site basis, $\tilde{c}_{n}(t-\tau, t)$, can be derived with the help of eq 21 at time $t-\tau$, together with eq 22:

$$
\begin{aligned}
\tilde{c}_{n}(t-\tau, t) & =\sum_{\alpha}\left\langle n \mid \Phi_{\alpha}(t-\tau)\right\rangle e^{i \epsilon_{\alpha} \tau / \hbar} c_{\alpha}(t) \\
& =\sum_{\alpha k} e^{i\left(\epsilon_{\alpha} / \hbar+k \Omega\right) \tau} e^{-i k \Omega t}\left\langle n \mid \Phi_{\alpha, k}\right\rangle c_{\alpha}(t)
\end{aligned}
$$

Equations 22 and 24 consequently allow to express the interaction picture operator $\tilde{\mathrm{c}}_{1}^{\dagger}(t-\tau, t)$, appearing in the current formula (eq 13), via $\mathrm{c}_{\alpha}(t)$, dressed by exponential prefactors.

This spectral decomposition allows one to carry out the time and energy integrals in eq 13 for the net current entering the wire from the left lead. Thus, we obtain

$$
I_{L}(t)=\sum_{k} e^{-i k \Omega t} I_{L}^{k}
$$

with the corresponding Fourier components

$I_{L}^{k}=e \Gamma_{L}\left[\sum_{\alpha \beta k^{\prime} k^{\prime \prime}}\left\langle\Phi_{\alpha, k^{\prime}+k^{\prime \prime}} \mid 1\right\rangle\left\langle 1 \mid \Phi_{\beta, k+k^{\prime \prime}}\right\rangle R_{\alpha \beta, k^{\prime}}\right.$

$$
\begin{aligned}
& -\frac{1}{2} \sum_{\alpha k^{\prime}}\left(\left\langle\Phi_{\alpha, k^{\prime}} \mid 1\right\rangle\left\langle 1 \mid \Phi_{\alpha, k+k^{\prime}}\right\rangle+\left\langle\Phi_{\alpha, k^{\prime}-k} \mid 1\right\rangle\left\langle 1 \mid \Phi_{\alpha, k^{\prime}}\right\rangle\right) \\
& \left.f\left(\epsilon_{\alpha, k^{\prime}}-\mu_{L}\right)\right]
\end{aligned}
$$

Here, we have introduced the expectation values

$$
\begin{aligned}
R_{\alpha \beta}(t) & =\left\langle c_{\alpha}^{\dagger}(t) c_{\beta}(t)\right\rangle=R_{\beta \alpha}^{*}(t) \\
& =\sum_{k} e^{-i k \Omega t} R_{\alpha \beta, k}
\end{aligned}
$$

The Fourier decomposition in the last line is possible because all $R_{\alpha \beta}(t)$ are expectation values of a linear, dissipative, periodically-driven system and therefore 
share in the long-time limit the time-periodicity of the driving field. In the subspace of a single electron, $R_{\alpha \beta}$ reduces to the density matrix in the basis of the Floquet states, which has been used to describe dissipative, periodically-driven quantum systems in refs 16,18,24-27.

The next step towards the stationary current is to find the Fourier coefficients $R_{\alpha \beta, k}$ at asymptotic times. To this end, we derive from the equation of motion (10) a master equation for $R_{\alpha \beta}(t)$. Since all coefficients of this master equation, as well as its asymptotic solution, are $\mathcal{T}$-periodic, we can split it into its Fourier components. Finally, we obtain for the $R_{\alpha \beta, k}$ the inhomogeneous set of equations

$$
\begin{aligned}
& \frac{i}{\hbar}\left(\epsilon_{\alpha}-\epsilon_{\beta}+k \hbar \Omega\right) R_{\alpha \beta, k}= \\
& \frac{\Gamma_{L}}{2} \sum_{k^{\prime}}\left(\sum_{\beta^{\prime} k^{\prime \prime}}\left\langle\Phi_{\beta, k^{\prime}+k^{\prime \prime}} \mid 1\right\rangle\left\langle 1 \mid \Phi_{\beta^{\prime}, k+k^{\prime \prime}}\right\rangle R_{\alpha \beta^{\prime}, k^{\prime}}\right. \\
& \quad+\sum_{\alpha^{\prime} k^{\prime \prime}}\left\langle\Phi_{\alpha^{\prime}, k^{\prime}+k^{\prime \prime}} \mid 1\right\rangle\left\langle 1 \mid \Phi_{\alpha, k+k^{\prime \prime}}\right\rangle R_{\alpha^{\prime} \beta, k^{\prime}} \\
& \quad-\left\langle\Phi_{\beta, k^{\prime}-k} \mid 1\right\rangle\left\langle 1 \mid \Phi_{\alpha, k^{\prime}}\right\rangle f\left(\epsilon_{\alpha, k^{\prime}}-\mu_{L}\right) \\
& \left.\quad-\left\langle\Phi_{\beta, k^{\prime}} \mid 1\right\rangle\left\langle 1 \mid \Phi_{\alpha, k^{\prime}+k}\right\rangle f\left(\epsilon_{\beta, k^{\prime}}-\mu_{L}\right)\right)
\end{aligned}
$$

+ same terms with the replacement

$$
\left\{\Gamma_{L}, \mu_{L},|1\rangle\langle 1|\right\} \rightarrow\left\{\Gamma_{R}, \mu_{R},|N\rangle\langle N|\right\}
$$

For a consistent Floquet description, the current formula together with the master equation must obey class invariance. Indeed, the simultaneous transformation with eq 18 of both the master equation (eq 29) and the current formula (eq 26) amounts to a mere shift of summation indices and thus leaves the current, as a physical quantity, unchanged.

For the typical parameter values used below, a large number of sidebands contributes significantly to the Fourier decomposition of the Floquet modes $\left|\Phi_{\alpha}(t)\right\rangle$. Numerical convergence for the solution of the master equation (eq 29), however, is already obtained by just using a few sidebands for the decomposition of $R_{\alpha \beta}(t)$. This keeps the numerical effort relatively small and justifies a posteriori the use of the Floquet representation (eq 21). Yet we are able to treat the problem beyond the rotating-wave-approximation.

\subsection{Time-Averaged Current through the Molecular Wire}

Equation 25 implies that the current $I_{L}(t)$ obeys the time-periodicity of the driving field. Since we consider here excitations by a laser field, the corresponding driving frequency lies in the optical or infrared spectral range. In an experiment thus one will only be able to measure the time-average of the current. For the net current entering through the left contact, it is given by

$$
\begin{aligned}
& \bar{I}_{L}=I_{L}^{0}= \\
& e \Gamma_{L} \sum_{\alpha k}\left[\sum_{\beta k^{\prime}}\left\langle\Phi_{\alpha, k^{\prime}+k} \mid 1\right\rangle\left\langle 1 \mid \Phi_{\beta, k^{\prime}}\right\rangle R_{\alpha \beta, k}\right. \\
& \left.-\left\langle\Phi_{\alpha, k} \mid 1\right\rangle\left\langle 1 \mid \Phi_{\alpha, k}\right\rangle f\left(\epsilon_{\alpha, k}-\mu_{L}\right)\right]
\end{aligned}
$$

By replacing $\{|1\rangle, L\} \rightarrow\{|N\rangle, R\}$, one obtains the current that enters from the right, $I_{R}(t)$, and the corresponding Fourier coefficients and time averages.

Total charge conservation of the original wire-lead Hamiltonian (eq 1) of course requires that the charge on the wire can only change by current flow, amounting to the continuity equation $\dot{Q}_{\text {wire }}(t)=I_{L}(t)+I_{R}(t)$. Since asymptotically the charge on the wire obeys, at most, the periodic time-dependence of the driving field, the time-average of $\dot{Q}_{\text {wire }}(t)$ must vanish in the long-time limit. From the continuity equation, one then finds that $\bar{I}_{L}+\bar{I}_{R}=0$, and we can introduce the time-averaged current

$$
\bar{I}=\bar{I}_{L}=-\bar{I}_{R}
$$

This continuity equation can be obtained directly from the average current formula (eq 30) together with the master equation (eq 29), as has been explicitly shown in ref 8 .

\section{LASER-ENHANCED CURRENT}

\subsection{Bridged Molecular Wire}

As a working model, we consider a molecule consisting of a donor and an acceptor site and $N-2$ sites in between (cf. Fig. 1). Each of the $N$ sites is coupled to its nearest neighbors by a hopping matrix element $\Delta$. The laser field renders each level oscillating in time with a position-dependent amplitude. Thus, the corresponding time-dependent wire Hamiltonian is

$$
\begin{aligned}
H_{n n^{\prime}}(t)= & -\Delta\left(\delta_{n, n^{\prime}+1}+\delta_{n+1, n^{\prime}}\right) \\
& +\left[E_{n}-A x_{n} \cos (\Omega t)\right] \delta_{n n^{\prime}}
\end{aligned}
$$

where $x_{n}=(N+1-2 n) / 2$ is the scaled position of site $|n\rangle$. The energy $A$ equals the electron charge multiplied by the electrical field amplitude of the laser and the distance between two neighboring sites. The energies of the donor and the acceptor orbitals are assumed to be at the level of the chemical potentials of the attached leads, $E_{1}=E_{N}=\mu_{L}=\mu_{R}$. The bridge levels $E_{N}, n=2, \ldots, N-1$, lie $E_{B} \gg \Delta$ above the chemical potential, as sketched in Fig. 1. 
In all numerical studies, we will use a symmetric coupling, $\Gamma_{L}=\Gamma_{R}=\Gamma$. The hopping matrix element $\Delta$ serves as the energy unit; in a realistic wire molecule, $\Delta$ is of the order $0.1 \mathrm{eV}$. Thus, our chosen wire-lead hopping rate $\Gamma=0.1 \Delta / \hbar$ yields $e \Gamma=2.56 \times 10^{-5}$ Ampère and $\Omega \approx 10 \Delta / \hbar$ corresponds to a laser frequency in the near infrared. For a typical distance of $5 \AA$ between two neighboring sites, a driving amplitude $A=\Delta$ is equivalent to an electrical field strength of $2 \times 10^{6} \mathrm{~V} / \mathrm{cm}$.

\subsection{Average Current at Resonant Excitations}

Let us first discuss the static problem in the absence of the field, i.e., for $A=0$. In the present case, where the coupling between two neighboring sites is much weaker than the bridge energy, $\Delta<<E_{B}$, one finds two types of eigenstates: One forms a doublet whose states are approximately given by $(|1\rangle \pm|N\rangle) / \sqrt{2}$. Its splitting can be estimated in a perturbational approach ${ }^{28}$ and is approximately given by $2 \Delta\left(\Delta / E_{B}\right)^{N-2}$. A second group of states is located on the bridge. It consists of $N-2$ levels with energies in the range $\left[E_{B}-2 \Delta, E_{B}+2 \Delta\right]$. In the absence of the driving field, these bridge states mediate the super-exchange between the donor and the acceptor. This yields an exponentially decaying length dependence of the conductance. ${ }^{3,10}$

This behavior changes significantly when a driving field with a frequency of $\Omega \approx E_{B} / \hbar$ is switched on. Then the resonant bridge levels merge with the donor and the acceptor state to form a Floquet state. This opens a direct channel for the transport, resulting in an enhancement of the electron current, as depicted in Fig. 2 where we plot the current amplification, defined as the ratio of the time-averaged current to the current in the absence of the laser, $\eta=\bar{I} / I_{0}$ : In a wire with $N=8$ sites, one finds peaks in the current when the driving frequency

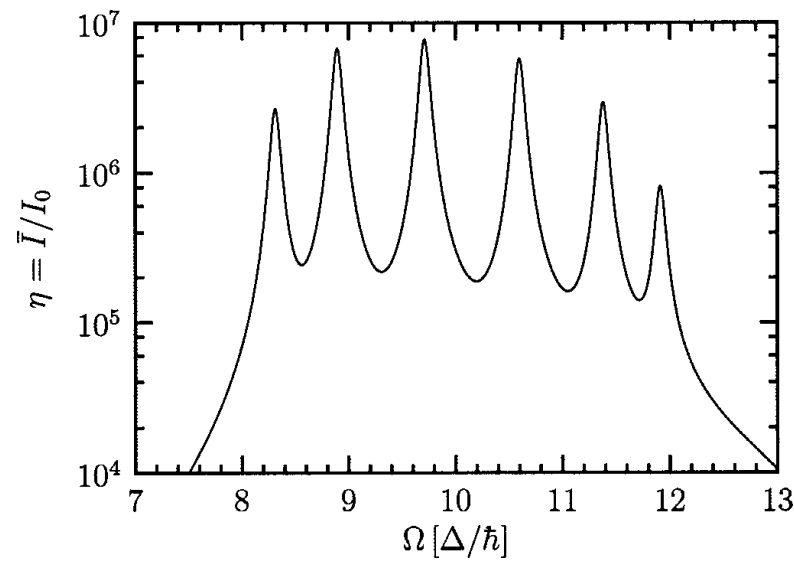

Fig. 2. Amplification of the time-averaged current through the wire sketched in Fig. 1 with $E_{B}=10 \Delta$. The scaled amplitude is $A=0.1 \Delta$; the applied voltage $\mu_{L}-\mu_{R}=5 \Delta / e$. The other parameters are $\Gamma=\Gamma_{L}=\Gamma_{R}=0.1 \Delta / \hbar, k_{B} T=0.25 \Delta$. matches the energy difference between the donor/acceptor doublet and one of the $N-2=6$ bridge levels. The voltage applied is always so small that the bridge levels lie above the chemical potentials of the leads. The amplification can assume many orders of magnitude, cf. Fig. 2. Generally, the response of a system to weak resonant driving scales with the damping and the driving amplitude. Figure 3 demonstrates this behavior for the peaks of the electrical current. The peak heights at the maxima of the time-averaged current are found to be proportional to $A^{2} / \Gamma$. Further scaling behavior is found for the current peaks as a function of the wire length: The average current no longer exhibits the exponentially decaying length dependence that has been found for bridged super-exchange. By contrast, it emerges proportional to $1 /(N-1)$. This can be appreciated in Fig. 4,

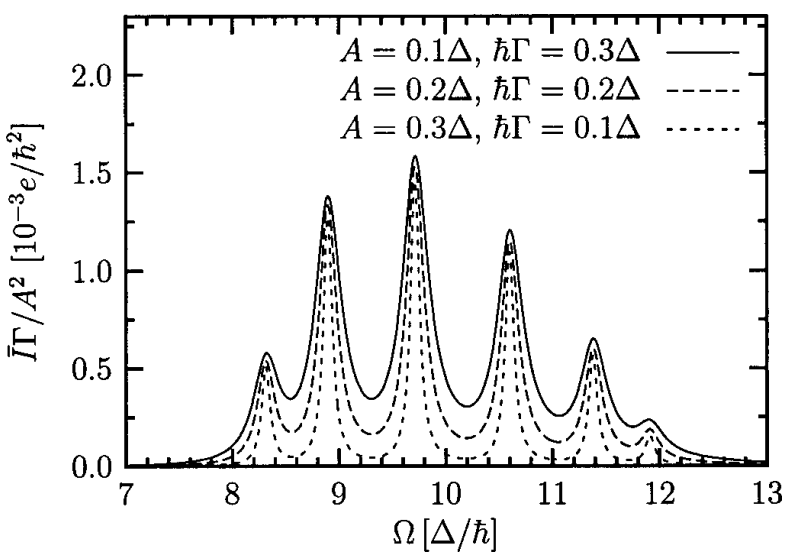

Fig. 3. Average current $\bar{I}$ as a function of driving frequency $\Omega$ for various driving amplitudes $A$ and coupling strength $\Gamma=\Gamma_{L}=\Gamma_{R}$. All other parameters are as in Fig. 2.

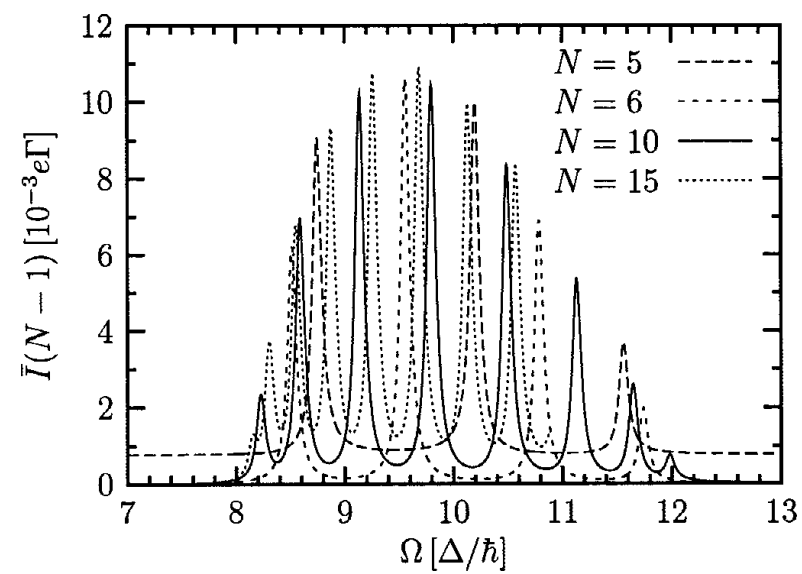

Fig. 4. Average current $\bar{I}$ as a function of driving frequency $\Omega$ for various wire length $N$. All other parameters are as in Fig. 2. 
where the scale of the abscissa is chosen proportional to $N-1$ such that it suggests a common envelope function. Put differently, the current is essentially inversely proportional to the length, as in the case of Ohmic conductance.

In summary, we find current peaks whose height $\bar{I}_{\text {peak }}$ scales according to

$$
\bar{I}_{\text {peak }} \propto \frac{A^{2}}{(N-1) \Gamma}
$$

Thus, the current, especially for long wires, is much larger than the corresponding current in the absence of the driving field.

\section{CONCLUSIONS}

We have presented a detailed derivation of the Floquet transport formalism that has been applied in refs 7-9. The analysis of a bridged molecular wire revealed that resonant excitations from the levels that connect the molecule to the external leads to bridge levels yield peaks in the current as a function of the driving frequency. In a regime with weak driving and weak electron-lead coupling, $\Delta \gg \Gamma, A$, the peak heights scale with the coupling strength, the driving amplitude, and the wire length. The laser irradiation induces a large current enhancement of several orders of magnitude. The observation of these resonances could serve as an experimental starting point for the more challenging attempt of measuring quantum ratchet effects ${ }^{7,8}$ or current switching by laser fields. ${ }^{9}$

Acknowledgments. This work has been supported by SFB 486 and by the Volkswagen-Stiftung under grant No. I/77 217. One of us (S.C.) has been supported by a European Community Marie Curie Fellowship.

\section{REFERENCES AND NOTES}

(1) Aviram, A.; Ratner, M.A. Chem. Phys. Lett. 1974, 29, 277.

(2) Joachim, C.; Gimzewski, J.K.; Aviram, A. Nature 2000, 408, 541.

(3) Nitzan, A. Annu. Rev. Phys. Chem. 2001, 52, 681.

(4) Hänggi, P.; Ratner, M.; Yaliraki, S. Special Issue: Processes in Molecular Wires. Chem. Phys. 2002, 281, 111-502.
(5) Cui, X.D.; Primak, A.; Zarate, X.; Tomfohr, J.; Sankey, O.F.; Moore, A.L.; Moore, T.A.; Gust, D.; Harris, G.; Lindsay, S.M. Science 2001, 294, 571.

(6) Reichert, J.; Ochs, R.; Beckmann, D.; Weber, H.B.; Mayor, M.; Löhneysen, H.v. Phys. Rev. Lett. 2002, 88, 176804.

(7) Lehmann, J.; Kohler, S.; Hänggi, P.; Nitzan, A. Phys. Rev. Lett. 2002, 88, 228305.

(8) Lehmann, J.; Kohler, S.; Hänggi, P.; Nitzan, A. J. Chem. Phys. 2003, 118, 3283.

(9) Lehmann, J.; Camalet, S.; Kohler, S.; Hänggi, P. Chem. Phys. Lett. 2003, 368, 282.

(10) Mujica, V.; Kemp, M.; Ratner, M.A. J. Chem. Phys. 1994, 101, 6849.

(11) Datta, S. Electronic Transport in Mesoscopic Systems. Cambridge University Press: Cambridge, 1995.

(12) Nitzan, A. J. Phys. Chem. 2001, A 105, 2677.

(13) Petrov, E.G.; Hänggi, P. Phys. Rev. Lett. 2001, 86, 2862.

(14) Lehmann, J.; Ingold, G.-L.; Hänggi, P. Chem. Phys. 2002, 281, 199.

(15) Manakov, N.L.; Ovsiannikov, V.D.; Rapoport, L.P. Phys. Rep. 1986, 141, 319.

(16) Grifoni, M.; Hänggi, P. Phys. Rep. 1998, 304, 229.

(17) Blümel, R.; Graham, R.; Sirko, L.; Smilansky, U.; Walther, H.; Yamada, K. Phys. Rev. Lett. 1989, 62, 341.

(18) Kohler, S.; Dittrich, T.; Hänggi, P. Phys. Rev. 1997, E 55,300 .

(19) Tikhonov, A.; Coalson, R.D.; Dahnovsky, Y. J. Chem. Phys. 2002, 116, 10909.

(20) Shirley, J.H. Phys. Rev. 1965, 138, B979.

(21) Sambe, H. Phys. Rev. 1973, A 7, 2203.

(22) Fainshtein, A.G.; Manakov, N.L.; Rapoport, L.P. J. Phys. 1978; B 11, 2561.

(23) Hänggi, P. In Quantum Transport and Dissipation; Dittrich, T.; Hänggi, P.; Ingold, G.L.; Kramer, B.; Schön, G.; Zwerger, W., Eds.; Wiley-VCH, Weinheim, 1998.

(24) Blümel, R.; Buchleitner, A.; Graham, R.; Sirko, L.; Smilansky, U.; Walter, H. Phys. Rev. 1991, A 44, 4521.

(25) Dittrich, T.; Oelschlägel, B.; Hänggi, P. Europhys. Lett. 1993, 22, 5.

(26) Kohler, S.; Utermann, R.; Hänggi, P.; Dittrich, T. Phys. Rev. 1998, E 58, 7219.

(27) Hänggi, P.; Kohler, S.; Dittrich, T. In Statistical and Dynamical Aspects of Mesoscopic Systems, Vol. 547 of Lecture Notes in Physics. Reguera, D.; Platero, G.; Bonilla, L.L.; Rub, J.M., Eds.; Springer: Berlin, 2000; pp 125-157.

(28) Ratner, M.A. J. Phys. Chem. 1990, 94, 4877. 